\title{
Investigation of Solid-state Chemical Lithiation of Single Crystalline Silicon Thin Window Anodes by Analytical Scanning and Transmission Electron Microscopy
}

Vladimir Oleshko ${ }^{1}$, Saya Takeuchi ${ }^{2}$ and Emily Bittle ${ }^{1}$

${ }^{1}$ NIST, Gaithersburg, Maryland, United States, ${ }^{2}$ Theiss Research, La Jolla, California, United States

Silicon is regarded as one of the most promising anode materials for high-energy density Li-ion batteries because of its exceptional theoretical specific capacity of $4200 \mathrm{mAh}$ g-1, which is more than 10 times the capacity of graphite $\left(372 \mathrm{mAh} / \mathrm{g}^{-1}\right)$ [1,2]. However, alloying silicon with lithium is fundamentally different from classical Li intercalation into graphite. Significant microstructural changes and volume expansion up to $400 \%$ during alloying can cause degradation, pulverization, loss of electrical contact and consequently rapid capacity fading [1,2]. Many studies have attempted to address these issues via nanoscale structural modification of anodes using high surface-to-volume ratio nanostructured Si that better accommodates strain, while allowing shorter diffusion lengths for $\mathrm{Li}$ ions and faster charge/discharge rates. In recent years, stable specific capacities over $1200 \mathrm{mAhg}-1$ for more than 1000 cycles have been realized with nanostructured Si-based anodes, suggesting a great potential for application in batteries [2]. Affirmative description of lithium kinetics and understanding of its evolution mechanisms in Si nanostructures are still quite limited [3]. In this work, we report on the solid-state lithiation of electron transparent single crystalline c-Si anodes by thermal evaporation of metal lithium foil in a vacuum. The average thickness of evaporated films was monitored by controlling evaporation rates of Li. We used pdoped $35 \mathrm{~nm}$-thick window < $100>$-oriented single crystalline planar c-Si membranes as a planar model system suitable for characterization with high-resolution analytical probe-corrected scanning and transmission electron microscopy (S/TEM). Such direct solid-state lithiation is a dry process without complications caused by side reactions under wet electrochemical processing. After lithiation, the membranes were transferred for examination in a S/TEM using a vacuum transfer TEM holder to prevent the samples from being exposed to an ambient environment. Controlled-dose bright/dark-field (BF-/DF)TEM, selected-area electron diffraction (SAED), high-resolution TEM (HRTEM), STEM, and electron energy-loss spectroscopy (EELS) were employed to characterize the morphology, crystallinity and chemical compositions of the lithiated membranes (Figs. 1 and 2). In the absence of an applied external electric field, lithiation of a $<100>$ - single crystalline c-Si membranes is driven primarily by the concentration gradient and occurs as a fast Li-consuming chemical process, closely analogous to diffusive solid-state amorphization [4]. Determining local phase and chemical compositions of the samples from analyses of low-loss and core-loss EEL spectra (Fig. 2), we found highlylithiated metastable Li-Si glass with an atomic ratio of $\mathrm{Li} / \mathrm{Si} \sim 2.2$, close to a $\mathrm{Li}_{7} \mathrm{Si}_{3}$ phase [5], that could coexist with c-Si since the crystallization of equilibrium intermetallic phases is frustrated during lithiation at room temperature. 


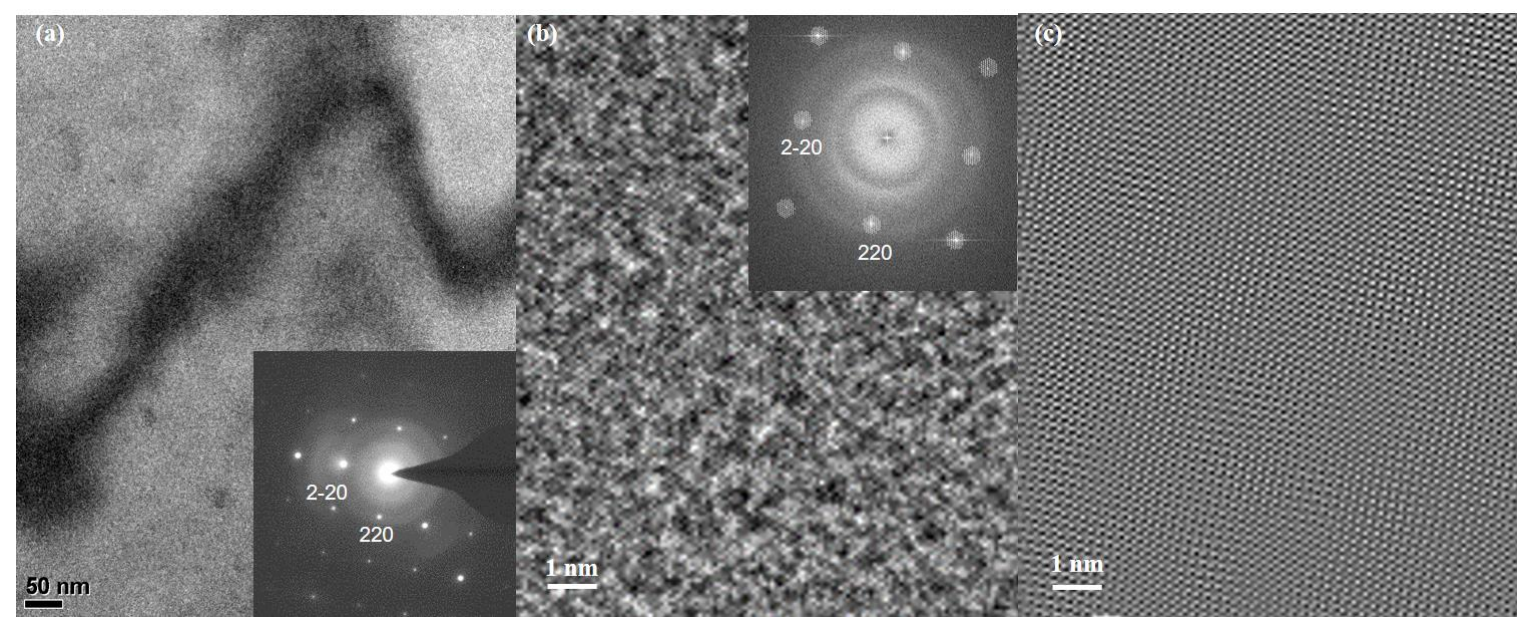

Figure 1. Chemically lithiated $35 \mathrm{~nm}$-thick window Si membrane with a $38 \mathrm{~nm}$ evaporated Li film: (a) BF-TEM, bending contours from the remaining c-Si phase. SAED pattern (bottom inset) shows diffuse rings from a Li-Si glass and point Bragg reflections from c-Si, near zone axis B = [001]. (b) HRTEM, barely visible lattice fringes through predominantly amorphous Li-Si glass. FFT pattern (top inset) reveals diffuse rings and point (220) reflections. (c) (220) c-Si lattice fringes distorted due to local strain were derived by Fourier-filtering from image (b) using spot reflections selected in the inset.

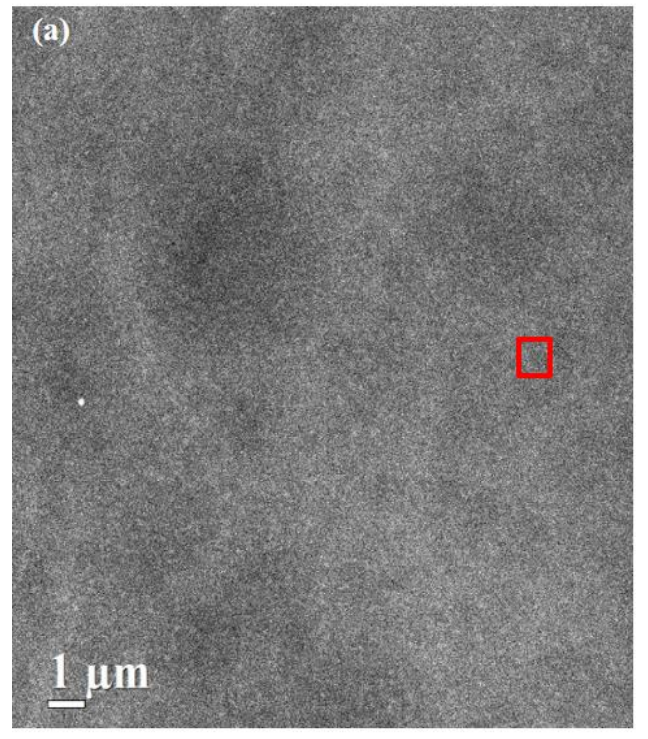

(b)

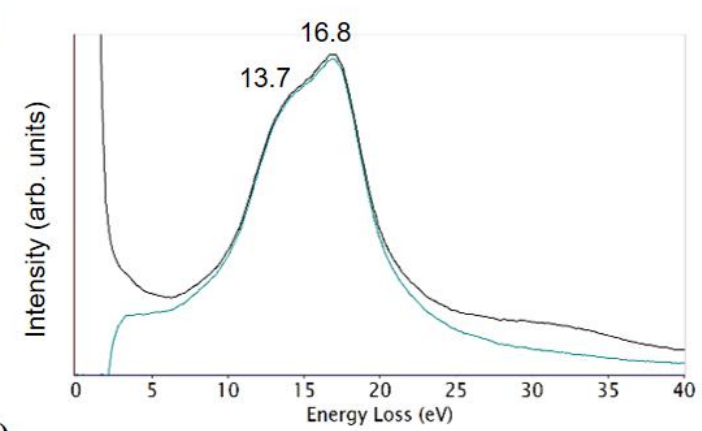

(c)

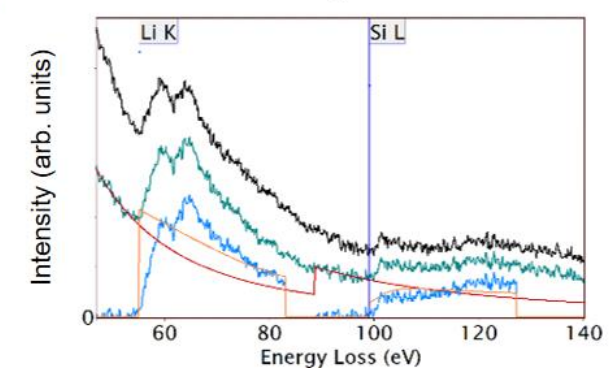

Figure 2. STEM-EELS of a lithiated Si membrane. (a) High-angle annular DF (HAADF) STEM, largearea view reveals weak contrast variations over a randomly distributed Li-Si glass film. (b) Low-loss EEL spectrum (black) acquired from the area marked by red box in (a) shows a shoulder at $13.7 \mathrm{eV}$ assigned to a Li7Si3 glass in addition to the bulk Si plasmon at $16.8 \mathrm{eV}$. A single scattering distribution (green) is derived by a Fourier-log deconvolution of the initial spectrum. (c) Core-loss spectrum from the same region with an estimated atomic ratio $\mathrm{Li} / \mathrm{Si}=2.2$. Fitted extrapolated power law backgrounds for the $\mathrm{LiK}$ and the SiL2,3 edges shown in red were used to extract the net edges (blue). The calculated Hartree-Slater scattering cross-sections for $\mathrm{Li}$ and $\mathrm{Si}$ are shown in orange. 


\section{References}

[1] VP Oleshko, et al, Microsc. Microanal. 22 (S3), (2016) 1556; ibid 24 (S1), (2018) 1480-1481.

[2] H Wu, et al, Nat. Nanotechnol. 4 (2012) 1943-1948.

[3] J-H Seo et al, RSC Adv., 5 (2015) 17548-17443.

[4] P Limthongkul, et al, Acta Mater. 51 (2003) 1103-1113.

[5] P Danet el al, Phys. Chem. Chem. Phys. 12 (2010) 220-226 